\title{
Die Resektion der Nasenscheidewand bei
} primärer Tuberculose.

Vou Prof. Dr. A. Ónodi in Bindapest.

Was die Therapie der primären tuberculösen Veränderungen der Nasenseheidewand betrifft, so sind wir immer anf Grund positiver histologiseher Diagnose für die endonasale Kurettage eingetreten, und auch nach Rezidiven haben wir gute Resultate erzielt. Auf die anderen Behandlungsverfahren werden wir noch zuräckkommen. Im Anschluli an einen aluỉerordentlich interessanten nnd Jehrreichen Fall bespreche ich die Frage der chirurgischen Behandlung der primiren Tuberculose der Nasenscheidewand. Den Fall macht auch der Lmstand wichtig, dal das klinische Bild und die mikroskopische Untersuchung des exzidierten Teiles fiir ein Carcinom der Scheidewand sprach, und auf dieser Grundlige ist auch der radikale chirurgische Eingriff vorgenommen worden. 
Paticnt J. II, 60 Jalıre alt, erschien bei nir Ende April 1904 mit Herrn Dr. Jannovitx. Nach seiner Aussage hatte er links vor einem Jahre eine geringe Nasenblutung, welche sich einige Male wiederbolte. Schmerzen hatte er nicht. Bei Gelegenheit eines unangenehmen Geftihls des Kratzens besichtigte er im Spiegel die Nase und sah in der Mittellinie eine kleine Erhobung. $E_{r}$ bekam eine Salbe, welche das Jucken zum Schwinden brachte. Die Rhinoskopie zeigte die rechte Nasenhalfte normal, auf der linken Seite zeigte die Nasenscheidewand eine auffallend große Veranderung. Das Bild machte mir den Eindruck einer bosartigen Geschwulst, ebenso auch vorher auf einen Fachkollegen, der den Kranken sah; die Abbildung zeigt den resezierten Teil der Nasenscheidewand mit der ganzen Ausbreitung der krankhaften Veränderung. Die später zu beschreibende Veränderung erstreckte sich zụm größten Teil auf den knorpeligen Teil der Scheidewand, der häutigè Teil der Scheidewand ist frei, beweglich, ihre Schleimhaut glatt, intakt. Aus einzelnen gröBeren, von gesunder Schleimhaut scharf umschriebenen, 5 mm hervorragenden Randteilen der Greschwulst lieB ich durch Herrn Dr. Jannovitz Stücke exzidieren. Die mikroskopische Untersuchung, die Herr Dozent B. Vas vornahm, ergab folgendes:

J.-No. 934 Budapest, 1904, 15. April. Der Schnitt des untersuchten Gewebes: in bindegewebigen Stroma eingelagerte, verschieden breite und lange Epithelzapfen, aus denen in das stellenweise mit Rundzellen stark infiltrierte Bindegewebe fadenförmige Epithelstränge hizeinragen, welche abweichend von den in den Epithelzapfen sich befindenden Epithelzellen, mit verschiedenen Kernteilungsformen einen Polymorphiamus zeigen. Nach meiner Meinung ist das exstirpierte Gebilde ein Papillom, an dessen eivzelnen Stellen die jungen Wucherungen die Tendenz einer Heterotopie besitzen, also in ein Carcinom überzugehen beginnen. Dieser Unstand muß für die Beurteilung des Falles und die weitere Behandlung maßgebend sein. Auf Grund dieses Befundes und des klinischen Bildes haben wir den radikalen Eingriff am 6. Mai ausgefiihrt.

Die Operation hat der Kranke mittels Lokalanasthesie gut vertragen, die Hant der Nase ist mit Aethylchlorid, die Nasenscheidewand mit Adrenalin und $20 \%$ Cocainlösung berührt worden. Die Nasenhöhle wurde in der Mittellinie eröffnet; in diesem so gewonnenen Raum zeigte sicb, daß außer den krankbaften Versinderungen der Nasenscheidewand oben, knapp am unteren Drittel der Nasenbeine, noch ein verborgener Herd, eine Granulationsmasse vorhanden ist. Aus diesem Gruude wurde der untere Teil des linken Nasenbeines entfernt und durch osteoplastische Resektion der Stirnfortsatz des Kieferbeines mit den Hautlappen aufgeklappt; so wurde die ganze linke Nasenböhle vollkommen übersichtlich. Nachdem der erwähnte latente Herd mit einem kleinen Knochenteil entfernt wurde, haben wir den knorpeligen Teil und einen Teil der knöchernen Scheidewand mit der gesunden Umgebung reseziert. Die Omgebung des gesunden Randes haben wir mit einem Thermokauter in der A usdehnung von $5-10 \mathrm{~mm}$ zerstört.

Nach Naht der Hautwunde, Tamponade und Ausspülung heilte die Wunde per primam, und der Patient verließ nach zehn Tagen das Spital. Den Kranken hatte ich nach einem Jahre gesehen, seine Nasenhöhle war ganz in Ordnung, die Rănder der Nasenscheidewand vernarbt, nur an Rande der Nasenöffnung zeigte sich eine kleino Veränderung, die mittels Korettage geheilt wurde. Von seiten der Lrunge waren weder vor der Operation noch nachher Veränderungen zu konstatieren.

Den resezierten Teil der Nasenscheidewand illustriert das Bild; die histologische Untersuchung besorgte Herr Entz, Assistent am Pathologischen Institut des Herrn Professor A. v. Genersich. Der resezierte Teil ist $38 \mathrm{~mm}$ lang, $30 \mathrm{~mm}$ breit und $12 \mathrm{~mm}$ dick, die geschwulstartig sich erhebende Schleimhaut ist unten und rückwärts glatt, feinhöckerig, hinten oben zeigt sie glatte, erbsengrobe Hocker und rorne eine $25 \mathrm{~mm}$ hohe und $10 \mathrm{~mm}$ breite Dlceration, mit unterminierten Rändern. Die Oberffäche wird von einem mehrschichtigen Plattenepithtl bedeckt, welches eine dünne Hornschicht zeigt, die Epithelschicht ist dick und reicht tief in die Ausführungsgänge der Drüsen. Das Bindegewebe der Drüsen in der Schleimhaut und die Submukosa ist mit Rundzellen infiltriert. Die Ausführungsgänge der Drüsen besitzen hohes Zylinderepithel. Zwischen der Schleimhaut und dem Perichondrium ist ein aus Follikeln bestehendes Gewebe vorhanden, welches durch bindegewebige Stränge in kleinere und größere Abschnitte geteilt wird. Die Rundzellen in den Follikeln sind verschieden groB und sehr chromatinrejch, nach innen folgen eosinophilartige und große flache epitheloide Zellen mit chromatinnetzigen Körnern. Stellenweise einige grobkörprige Riesenzellen mit vielen ovalen und rumden Kernen und feinem Chromatinnetz. Die Zellenmassen sind durchsat mit polynukleären Zellen. Eine Verkäsung oder Degeneration war nicht zu konstatieren.
Der Befund zejgt, dab es sich um eine Tuberculose der Nasenscheidewand handelte. Der erste Befund hat einen dem Krobs entsprechenden, radikalon Eingriff im Interesse des Kranken erfordert, auch sonst bestand die Indikation wegen der groben Ausbreitung des Tubereuloms und des erwähnten latenten Herdes. Wir erwähnen kurz, dab die ulcerierende Form an der Scheidewand seltener ist, öfter treffen wir Proliferationen und Tuberculome. Aehnliche Falle haben Fränkel, Chiari, Hasslauer, Onodi, Hajek, Silberstein, Grönbeck, Prota, Bar, Texier, Bronnor, Hins. berg, Thomas, Massei, Beck und Pasch veröffentlicht. Wir wollen die bekannten klinischen und diagnostischen Verhabltnisse nicht erörtern, sondern nur die Therapie der primären tuberculösen Veränderungen der Nasenscheidewand berühren. Die Behandiung besteht zumeist in der Entfernung und Kurettage mit Schlinge und scharfem Löffel der tuberculösen Granulationen. Dann folgt die Aetzung, die Behandlung mit Milchsäure. Uebrigens sind dio verschiedenen Verfahren der Aetzungen ohne chirurgische Eingriffe benutzt worden. Einige haben von der Milchsäure brfolge gesehen, andere haben sich vom Gegenteil überzeugt. Es ist zu bemerken, dafi nach energischen Auskratzungon und sorgfältiger Entfernung der krankhaften Verände. rungen wiederholt Rezidive beobachtet wurden. Bei diffusen taberculösen Infiltrationen und gröberen Ausdehnungen macht Pascl keine Exzisionen, sondern benutzt die Pyrogallussäure und empfiehlt sie, da sie günstig einwirkt, wenn sie auch den Prozeb nicht dauernd heilt. Die von Lermoyez und Mahu empiohlene Heißluftbehandlung ist beim Lupus angewendet worden, bei der 'Tuberculose noch nicht. Beck versuchte das Radium. Pasch vorsuchte dio Stauungstherapie in einigen Fällen ohne besonderen Erfolg.

In unseren Fällen haben wir auf Grund der histologischen Untersuchung immer die endonasale, chirurgische Entfernung ausgeführt (energische Auskratzung), und zwar meistens mit Erfolg; in einem kleineren. Teil der Fälle traten die Rezidive dennocli ein, sodaf der Eingriff wiederholt wurde. Pasch hemerkt zutreffend, daß bei der Frage der Rezidive nicht gerade die in der Tiefe zurückgelassenen bacillären Tuberkelherde, sondern vielmehr die latenten Initialherde eine Rolle spielen. Bei einem Rezidiv klappte er zwecks vollständiger Uebersichtlichkeit, ferner zu radikaler Entfernung dor krankhaften Verinderungen die Nasenhöhle in der Medianlinie auf. Der radikale Eingriff in unserem Falle ist günslig verlaufen unter den erwähnten Umständen, die Berechtigung hätte or auch übrigens in der Größe der tuberculösen Veränderung und in dem Vorhandensein eines latenten tuberculösen Herdes gefunden. Bei der Behandlung der primären Tuberculose der Nasenscheldewand, wenn die Nasenhöhle zu übersehen und die Ausdehnung klein ist, führen wir die endonasale chirurgische Entfernung aus; in jenen Fällen aber, wo die primäre Veränderung eine größere Ausdehmung hat und neben einer unvollständigen Uebersicht ein latenter Tuberkelherd nicht auszuschlieben ist, wie in unserem Falle zwar aus anderer Indikation, soll die Nasenhöhle in der Mittellinie eröffnet, wenn notwendig die osteoplastische Resektion des Nasenbeines und des Stirnfortsatzes des Oberkiefers und die Resektion des tuberculös veränderten Teiles der Nasenscheidewand ausgeführt werden. Die Aufklappung ermöglicht die Auffindung eines latenten Herdes, und die Resertion des Scheidewandteiles sichert die radikale Entfernung der krankhaften Veränderung. was von der einfachen Auskratzung nicht gesagt werden kann, denn bacilläre Herde können in der Tiefe zurückgelassen werden. Indem wir im Anschlub an unsern Fall auf dieses radikale Verfahren die Aufmerksamkeit lenken, für entsprechende Fälle der primären Tuberculose der Nasenscheidewand, halten wir den Fall auch für lehrreich wegen seiner klinischen Erscheinung, wegen seiner Indikation auf Grund des ersten histologischen Befundes und wegen der ausgeführten radikalen Operation. 